ORIGINAL ARTICLE

\title{
Pessary Expulsion Rate and Risk Factors for Expulsion in Southern Thai Women with Pelvic Organ Prolapse
}

\author{
Pukavut Withayajiakkhajorn ${ }^{1}$, Wongsakorn Limbutara ${ }^{2}$, Chatchawan Gorsagun ${ }^{3}$
}

\begin{abstract}
Objective: To investigate and ascertain the rate of and risk factors for vaginal pessary expulsion after vaginal pessary use among pelvic organ prolapse (POP) patients in Southern Thailand.

Materials and methods: The retrospective medical records' review and telephone interview were conducted in 140 patients with POP managed by vaginal pessary insertion as the first-line treatment during the period between March 2015 and January 2018. Factors influencing pessary expulsion, pessary discontinuation, and adverse event after pessary use were investigated.

Results: Of the 482 POP patients, vaginal pessaries were offered to 140 patients. The prolapse was in advanced stage (stage III-IV) in most of the patients $(77.1 \%)$, according to POP quantification (POP-Q) system. All of the pessaries offered were of ring type, without support in any prolapse stage. After vaginal pessary insertion, the expulsion rate was $22.1 \%$. The mean of the pessary continuation period in all patients were $20.5 \pm 8.2$ months. During the study period, the discontinuation rate was $22.8 \%$. Factors associated with pessary expulsion from logistic regression analysis were high body mass index [(BMI; relative risk (RR) 3.491, 95\% confidence interval (CI) $1.302-9.356 ; p=0.013]$; history of previous hysterectomy (RR 37.68, 95\% Cl 4.508-315.098; $p=0.001)$; age more than 65 years ( $\mathrm{RR} 3.71,95 \% \mathrm{Cl} 0.078-0.929 ; p=0.038)$; advanced degree of prolapse (RR 4.842, 95\% Cl 4.842-1.008; $p=0.049)$; and adverse effects related to pessary insertion were vaginal discharge (5\%), vaginal discomfort (13.5\%), vaginal erosion (7.1\%), vaginal bleeding (1.4\%), and constipation (0.7\%).

Conclusion: The expulsion and discontinuation rates of vaginal ring pessary in POP patients in any degree of prolapse were acceptable based on our experience from Southern Thailand. Factors associated with expulsion in this population were high BMI, history of previous hysterectomy, advanced age, and advanced degree of prolapse.
\end{abstract}

Keywords: Discontinuation, Expulsion, Pelvic organ prolapse, Vaginal pessary.

Journal of South Asian Federation of Menopause Societies (2019): 10.5005/jp-journals-10032-1184

\section{INTRODUCTION}

Pelvic organ prolapse (POP) is a condition that occurs following the loss of support to the uterus, which chronically affects women; ${ }^{1}$ this chronic dysfunction impacts nearly half of the women over the age of $50 .^{2}$ The prevalence of POP among postmenopausal women in Southeast Asian countries such as Thailand is similar to the global scale at $43.3 \%{ }^{3}$ Treatment options include both surgical and nonsurgical management. Surgical management consists of various methods with the concept mainly focusing on restoration of the defect site through abdominal, laparoscopic, or vaginal approaches. Conservative management mainly composed of lifestyle modification, pelvic floor muscle exercise, and vaginal pessaries. Pessaries are considered the first option in the United States ${ }^{4}$ and are commonly offered by gynecologists in most countries, including Thailand. Vaginal pessaries can be broadly categorized into two types as supportive and space-occupying pessaries. Supportivetype pessaries are generally recommended for symptomatic POP quantification (POP-Q) stages I and II, ${ }^{4}$ while space-filling pessaries are mostly prescribed for women with advanced prolapse. ${ }^{5}$

However, studies conducted in many countries including Thailand suggest the usefulness of support-type pessaries for treatment of both early and advanced stage prolapse. Advantages include convenient use, allowance of sexual activity, and minimal complications. ${ }^{6,7}$ Interestingly, these studies reported various reasons for the discontinuation of vaginal pessaries. Most authors agreed that the main reasons for pessary discontinuation were due to expulsion of the pessary ${ }^{7-9}$ but factors associated with pessary expulsion require further investigation.

\footnotetext{
${ }^{1-3}$ Department of Obstetrics and Gynecology, Hatyai Hospital, Hatyai, Songkhla, Thailand
}

Corresponding Author: Wongsakorn Limbutara, Department of Obstetrics and Gynecology, Hatyai Hospital, Hatyai, Songkhla, Thailand, Phone: +66 887906483, e-mail: Iwongsakorn@gmail.com

How to cite this article:Withayajiakkhajorn P, Limbutara W, Gorsagun C. Pessary Expulsion Rate and Risk Factors for Expulsion in Southern Thai Women with Pelvic Organ Prolapse. J South Asian Feder Menopause Soc 2019;7(2):59-61.

Source of support: Nil

Conflict of interest: None

Hence, the study's primary outcome was to investigate the rate of pessary expulsion 12 months after use and also determine the adverse effects and factors associated with the secondary outcomes of vaginal pessary expulsion and discontinuation.

\section{Materials and Methods}

Approval was obtained from Hatyai Hospital research ethical committee (protocol number 98/2560). A retrospective review of medical records and telephone interviews was done in 140 patients with POP using vaginal pessary inserted by gynecologist as the first-line treatment in Hatyai Hospital gynecology and urogynecology clinic between March 2015 and January 2018. All participants were followed for at least 12 months. Speculum examination, pelvic examination, and pessary maintenance review

() The Author(s). 2019 Open Access This article is distributed under the terms of the Creative Commons Attribution 4.0 International License (https://creativecommons. org/licenses/by-nc/4.0/), which permits unrestricted use, distribution, and non-commercial reproduction in any medium, provided you give appropriate credit to the original author(s) and the source, provide a link to the Creative Commons license, and indicate if changes were made. The Creative Commons Public Domain Dedication waiver (http://creativecommons.org/publicdomain/zero/1.0/) applies to the data made available in this article, unless otherwise stated. 
Flowchart 1: Study protocol

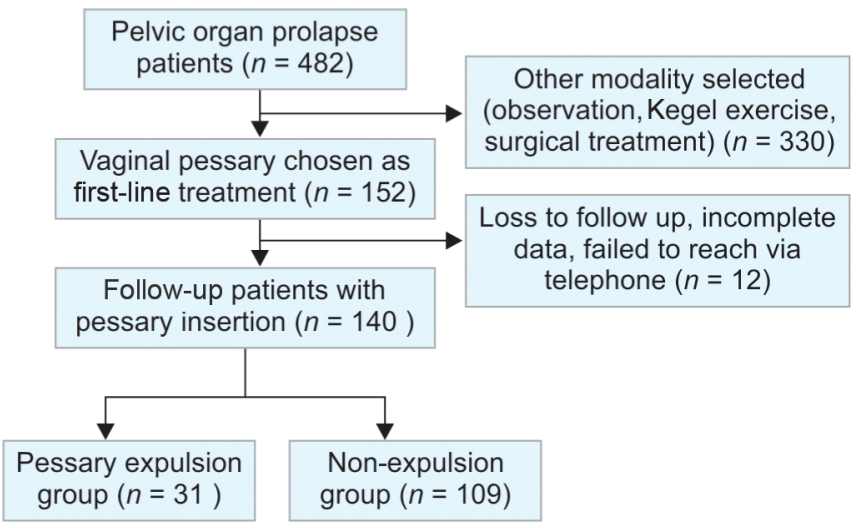

were performed by gynecologist at each visit to detect the possible complications such as vaginal discharge, vaginal bleeding, vaginal abrasion, or erosion.

Expulsion of the pessary was defined as pessary slipping out as reported by the patients at the follow-up period or during telephone interview. Discontinuation of the pessary was defined when patients desire to discontinue pessary or choose surgical management after using pessary (Flowchart 1).

Primary outcome is to determine the expulsion rate of vaginal pessaries and factors influencing pessary expulsion. Secondary outcomes are to determine pessary discontinuation rate and also the adverse events after using pessary such as vaginal discomfort, vaginal abrasion, vaginal discharge, vaginal bleeding, and constipation.

Demographic data were calculated using the mean for continuous variables. Categorical variables were calculated using Fisher's exact test and Mann-Whitney $U$ test. Univariate and multiple logistic regression analyses were applied to determine the factors influencing pessaries expulsion. When $p$ value $<0.05$ it was considered statistically significant, and statistical analysis was achieved using SPSS v.23.0.

\section{Results}

Vaginal pessaries were selected as the primary treatment by 140 of the 482 POP patients. Most (77.1\%) of the patients demonstrated advanced prolapse (stages III and IV). The mean age of the patients was 68.51 years $( \pm 8.81)$ and the mean parity was $4.4( \pm 2.3)$. Most of the patients (97.9\%) were postmenopausal. Thirty-five (24.8\%) patients had a history of surgery including total vaginal hysterectomy (6.4\%), vaginal hysterectomy (2.1\%), postpartum tubal resection (10\%), cesarean section (2.8\%), appendectomy $(2.1 \%)$, and inguinal hernia repair (1.4\%).

Expulsion rate after 12 months was $22.1 \%$, with the mean continuation period of $20.5 \pm 8.2$ months (Table 1). Pessary discontinuation was found in $22.8 \%$ after 12 months of use (Table 2 ). Factors associated with pessary expulsion are calculated by logistic regression analysis including high $\mathrm{BMI}$ (RR 3.491, 95\% Cl 1.302-9.356; $p=0.013)$, history of hysterectomy (RR $37.68,95 \% \mathrm{Cl} 4.508-315.098$; $p=0.001$ ), advanced age ( $>65$ years; RR $3.71,95 \% \mathrm{Cl} 0.078-$ $0.929 ; p=0.038$ ), and advanced prolapse (RR $4.842,95 \% \mathrm{Cl}$ 4.842-10.008; $p=0.049$ ) (Table 3). Adverse effects included vaginal discharge (5\%), vaginal abrasion (7.1\%), vaginal discomfort (13.5\%), bleeding per vagina (1.4\%), and constipation (0.7\%) (Table 4).
Table 1: Baseline characteristics

\begin{tabular}{ll}
\hline Characteristics $(n)$ & $n(\%)$ \\
\hline Mean age $( \pm$ SD) & $68.51 \pm 8.81$ \\
Mean BMI $( \pm$ SD) & $23.97 \pm 3.43$ \\
Menopausal status $(140)$ & $137(97.9)$ \\
Parity & \\
$\quad$ Nulliparous & $1(0.8)$ \\
$\quad$ Multiparous & $139(99.2)$ \\
Surgical history & \\
TAH \pm BSO & $9(6.4)$ \\
Vaginal hysterectomy & $3(2.1)$ \\
Tubal resection & $14(10)$ \\
Cesarean section & $4(2.8)$ \\
Appendectomy & $3(2.1)$ \\
Inguinal hernia repair & $2(1.4)$ \\
\hline
\end{tabular}

$\mathrm{TAH} \pm \mathrm{BSO}$, total abdominal hysterectomy with bilateral salpingooophorectomy

Table 2: Expulsion and discontinuation rates after 12 months of pessary use

\begin{tabular}{ll}
\hline & $n(\%)$ \\
\hline Expulsion rate & $7(22.1)$ \\
Discontinuation rate & $19(22.8)$ \\
\hline
\end{tabular}

Table 3: Multivariate analysis of the factors associated with the expulsion rate

\begin{tabular}{lcll}
\hline Factors & OR & $95 \% \mathrm{Cl}$ & p value \\
\hline High BMI (BMI > 25) & 3.491 & $1.302-9.356$ & 0.013 \\
$\begin{array}{l}\text { History of previous } \\
\text { hysterectomy }\end{array}$ & 37.68 & $4.508-315.098$ & 0.001 \\
$\begin{array}{l}\text { Age }>65 \text { years } \\
\begin{array}{l}\text { Advanced degree of } \\
\text { prolapse }\end{array}\end{array}$ & 3.71 & $0.078-0.929$ & 0.038 \\
\hline
\end{tabular}

Table 4: Adverse events after pessary use $(n=140)$

\begin{tabular}{lc}
\hline Adverse events & $n(\%)$ \\
\hline Vaginal discharge & $7(5)$ \\
Vaginal discomfort & $19(13.5)$ \\
Erosion & $10(7.1)$ \\
Vaginal bleeding & $2(1.4)$ \\
Constipation & $1(0.7)$ \\
\hline
\end{tabular}

\section{Discussion}

Pelvic organ prolapse is commonly encountered female, which increases as the population ages, with $43-76 \%$ of women showing loss of pelvic floor support during routine gynecological care. ${ }^{10-12}$ However, only $2.9-8.8 \%$ of women reported prolapse symptoms. ${ }^{1,11}$

Several treatment options are available for symptomatic POP, including lifestyle modification, Kegel exercises, mechanical devices, and reconstructive surgery. Despite its attractiveness, surgery has a known rate of reoperation at $10-30 \% .^{13,14}$ Therefore, many gynecologists offer conservative management as the first option.

Studies have reported high effectiveness of pessary use in the short-term, with success rates ranging from $41 \%$ to $74 \% .{ }^{5}$ Data from Thailand collected by Manchana et al. reported the successful use of ring pessaries for symptomatic patients, with $71 \%$ having POP-Q 
stages III and IV. The success rate in their study was 52\% at 13-month follow-up. ${ }^{7}$ In 2017, Yimphong et al. reported $86.4 \%$ success of pessary use over a period of 12 months. ${ }^{8}$ However, long-term data did not seem to show promising results, with the longest follow-up over 15 years showed that only $14 \%$ of patients continue to use pessary with a median of 7 years. ${ }^{10}$

Many authors attempted to determine the factors associated with vaginal pessary discontinuation, with results suggesting that one of the most common objective reasons for discontinuation was expulsion of the pessary. ${ }^{7-9}$ Yimphong et al. reported discontinuation rate of vaginal pessary use after 1 year at $13.5 \%$, with pessary expulsion rate at $26.3 \%$ as the main reason, concurring with our findings at an expulsion rate of $22.1 \%$. This may be due to the populations in both study were quite similar in terms of race and geographic variation. Regarding the history of previous hysterectomy, the findings of our study and that of Yimphong's concurred. It might be explained that shortening of vagina and narrowing of the apex following previous hysterectomy can exacerbate the difficulty of fitting procedure and promote expulsion leading to discontinuation. Similar results were also reported by Fernando et al. ${ }^{15}$ However, the correlation between high BMI and pessary expulsion in our findings contrasted with the results by Wang et al. ${ }^{16}$ We presumed the reason behind this finding was that populations with high BMI are also at risk of more advanced POP and tend to show higher stages of POP compared to the normal BMI populations, and this may affect their decision to more likely choose surgical intervention over conservative management due to the advanced degree of prolapse. ${ }^{16}$

Advanced age and advanced degree of prolapse were concomitantly found in most situations due to the degenerative nature of the pelvic organ supporting tissue promotes further degree of POP. Both of them were found to increase the risk of pessary expulsion in our study in contrast to the findings of Wolff et al. ${ }^{9}$ This could be from that the vaginal pessary is likely to be more difficult to retain its position in increased intra-abdominal pressure situation with regard to the advanced degree of prolapse.

Adverse events after vaginal pessary use in our study were recorded at $27.7 \%$ compared to the previous study results ranging from $56 \%$ to $58 \% .{ }^{10,17}$ Most commonly found adverse events were vaginal abrasion and vaginal infection. We found relatively lower rates of vaginal discharge (5\%) and vaginal bleeding (1.4\%) compared to previous studies in Thailand. ${ }^{8}$ Also, none of the women in our study presented major complications such as fistula, smallbowel obstruction, or sepsis.

To date, this study is the first in Southern Thailand to explore factors associated with pessary expulsion as the primary cause of discontinuation. Results will assist in identifying the risks associated with pessary expulsion and provide useful information for guiding patients in our clinical practice. Study limitations included data collection from single institution with limited sample size and retrospective nature of the data. Multicenter studies with larger sample sizes are required for future research. Our study's main focus was medium-term results of pessary use. Long-term follow-up is required for a more accurate assessment of the results.

In conclusion, the pessary expulsion and discontinuation rate of pessary in any degree of POP in our experience from Southern
Thailand is comparable to the global scale. Factors found to be associated with pessary expulsion in our study were high BMI, history of hysterectomy, advanced degree of prolapse, and advanced age.

\section{References}

1. Nygaard I, Barber MD, Burgio KL, et al. Prevalence of symptomatic pelvic floor disorders in US women. JAMA 2008;300(11):1311-1316. DOI: 10.1001/jama.300.11.1311.

2. Subak LL, Waetjen LE, Van den Eaden S, et al. Cost of pelvic organ prolapse surgery in the United States. Obstet Gynecol 2001;98(4):646-651. DOI: 10.1016/s0029-7844(01)01472-7.

3. Chuenchompoonut V, Bunyavejchevin S, Wisawasukmongchol W, et al. Prevalence of genital prolapse in thai menopausal women (using new standardization classification). J Med Assoc Thai 2005;88(1):1-4.

4. Cundiff GW, Weidner AC, Visco AG, et al. A survey of pessary use by members of the American Urogynecologic Society. Obstet Gynecol 2000;95(6, Part 1):931-935. DOI: 10.1097/00006250-20000600000029.

5. Jones KA, Harmanli OZ. Pessary use in pelvic organ prolapse and urinary incontinence. Rev Obstet Gynecol 2010;3(1):3-9. DOI: 10.3909/ riog0110.

6. Ding J, Chen C, Song XC, et al. Successful use of ring pessary with support for advanced pelvic organ prolapse. Int Urogynecol J 2015;26(10):1517-1523. DOI: 10.1007/ s00192-015-2738-1.

7. Manchana T. Ring pessary for all pelvic organ prolapse. Arch Gynecol Obstet 2011;284(2):391-395. DOI: 10.1007/s00404-010-1675-y.

8. Yimphong T, Temtanakitpaisan T, Buppasiri P, et al. Discontinuation rate and adverse events after 1 year of vaginal pessary use in women with pelvic organ prolapse. Int Urogynecol J 2017;;29(8):1123-1128. DOI: 10.1007/s00192-017-3445-x.

9. Wolff B, Williams K, Winkler A, et al. Pessary typesand discontinuation rates in patients with advanced pelvic organ prolapse. Int Urogynecol J 2017;8(7):993-997. DOI: 10.1007/ s00192-016-3228-9.

10. Sarma $\mathrm{S}$, Ying T, Moore $\mathrm{KH}$. Long-term vaginal ring pessary use: discontinuation rates and adverse events. BJOG 2009;116(13): 1715-1721. DOI: 10.1111/j.1471-0528.2009.02380.x.

11. MacLennan AH, Taylor AW, Wilson DH, et al. The prevalence of pelvic floor disorders and their relationship to gender, age, parity and mode of delivery. BJOG: Int J Obstet Gynaecol 2000;107(12):1460-1470. DOI: 10.1111/j.1471-0528.2000.tb11669.x.

12. Swift S, Woodman $P, O^{\prime} B o y l e ~ A$, et al. Pelvic organ support study (POSST): the distribution, clinical definition, and epidemiological condition of pelvic organ support defects. Am J Obstet Gynecol 2005;192(3):795-806. DOI: 10.1016/j.ajog.2004.10.602.

13. Olsen AL, Smith VJ, Bergstrom JO, et al. Epidemiology of surgically managed pelvic organ prolapse and urinary incontinence. Obstet Gynecol 1997;89(4):501-506. DOI: 10.1016/S0029-7844(97)00058-6.

14. Price N, Slack A, Jwarah $E$, et al. The incidence of reoperation for surgically treated pelvic organ prolapse: an 11-year experience. Menopause Int 2008;14(4):145-148. DOI: 10.1258/mi.2008. 008029.

15. Fernando RJ, Thakar R, Sultan AH, et al. Effect of vaginal pessaries on symptoms associated with pelvic organ pro-lapse. Obstet Gynecol 2006;108(1):93-99. DOI: 10.1097/01.AOG. 0000222903.38684.cc.

16. Wang Y, Yang J, Han J, et al. Factors influencing therapy decision in patients with severe pelvic organ prolapse. Zhonghua Fu Chan Ke Za Zhi 2015;50(2):112-115.

17. Cheung RY, Lee JH, Lee LL, et al. Vaginal pessary in women with symptomatic pelvic organ prolapse: a randomized controlled trial. Obstet Gynecol 2016;128(1):73-80. DOI: https://doi.org/10.1097/ AOG.0000000000001489. 